\title{
Fast, Quantitative, and Nondestructive Evaluation of Hydrided LWR Fuel Cladding by Small Angle Incoherent Neutron Scattering of Hydrogen
}

\author{
Y. Yan, ${ }^{1}$ S. Qian, ${ }^{1}$ K. Littrell, ${ }^{1}$ C. M. Parish,${ }^{1}$ and L. K. Plummer ${ }^{2}$ \\ ${ }^{1}$ Oak Ridge National Laboratory, Oak Ridge, TN 37831, U.S.A. \\ ${ }^{2}$ University of Oregon, Eugene, OR 97403, U.S.A.
}

\begin{abstract}
A nondestructive neutron scattering method to precisely measure the uptake of hydrogen and the distribution of hydride precipitates in light water reactor (LWR) fuel cladding was developed. Zircaloy-4 cladding used in commercial LWRs was used to produce hydrided specimens. The hydriding apparatus consists of a closed stainless-steel vessel that contains $\mathrm{Zr}$ alloy specimens and hydrogen gas. Following hydrogen charging, the hydrogen content of the hydrided specimens was measured using the vacuum hot extraction method, by which the samples with desired hydrogen concentrations were selected for the neutron study. Optical microscopy shows that our hydriding procedure results in uniform distribution of circumferential hydrides across the wall thickness. Small angle neutron incoherent scattering was performed in the High Flux Isotope Reactor at Oak Ridge National Laboratory. Our study demonstrates that the hydrogen in commercial Zircaloy-4 cladding can be measured very accurately in minutes by this nondestructive method over a wide range of hydrogen concentrations from a very small amount $(\approx 20 \mathrm{ppm})$ to over $1000 \mathrm{ppm}$. The hydrogen distribution in a tube sample was obtained by scaling the neutron scattering rate with a factor determined by a calibration process using standard, destructive direct chemical analysis methods on the specimens. This scale factor can be used in future tests with unknown hydrogen concentrations, thus providing a nondestructive method for determining absolute hydrogen concentrations.
\end{abstract}




\section{INTRODUCTION}

Zirconium alloys have been used in light water reactors (LWRs) for the past several decades. However, hydrogen uptake by these alloys increases with fuel burnup, causing the cladding ductility and failure energy to decrease. A very small amount of hydrogen can dramatically adversely affect materials performance $[1,2]$. A major area of focus for zirconium alloy cladding research has been on decreasing the waterside corrosion rate and hydrogen pick-up under normal operating conditions to increase the operational economy and safety margin [3, 4]. Recently, hydrogen embrittlement of zirconium cladding under loss of coolant accident (LOCA) conditions has been studied extensively [5-12], particularly because of the Fukushima accident.

Traditionally, the hydrogen content in zirconium alloys is measured using a high-temperature extraction method such as vacuum hot extraction (VHE). While this method has good accuracy for small amounts of hydrogen, it is destructive (the sample has to be melted during analysis). Since hydrogen has a large total neutron cross-section, including a large incoherent scattering cross-section, compared to zirconium and other metallic elements, neutron methods are an attractive option for nondestructive analysis. It has been demonstrated previously that neutron radiography and tomography can be used to study hydrogen distribution nondestructively using the property of neutron attenuation due to absorption and scattering from hydrogen to quantitatively determine the hydrogen content in samples with hydrogen concentrations higher than a few hundreds of parts per million [13-16].

The small angle incoherent neutron scattering (SAINS) method described here takes advantage of the fact that the largest component of the neutron cross-section for hydrogen is the inelastic incoherent cross-section, a process by which neutrons are scattered evenly in all directions. In SAINS, a large solid angle section of this approximately spherically symmetric scattering proportional to the hydrogen content in the illuminated sample volume is captured, giving much higher sensitivity to hydrogen than in the attenuation techniques but limited to a coarser area resolution. The results of this nondestructive method are compared to results from measurements of hydrogen concentrations using standard, destructive, direct chemical analysis by the high temperature extraction method for samples with a broad range of hydrogen concentrations. Correlations between the SAINS counts and VHE hydrogen contents in hydrided Zircaloy-4 cladding are established. The present work shows that a wide range of hydrogen content in commercial Zircaloy-4 fuel cladding can be quantitatively determined by the SAINS method, extending the range of nondestructive analysis to samples with orders of magnitude lower hydrogen concentrations than are typically measurable by neutron imaging techniques.

\section{Material and Experimental Procedure}

The materials examined for this work were sections of Zircaloy-4 cladding used in commercial pressurized water reactor (PWR) with an outside diameter of $9.5 \mathrm{~mm}$ and a wall thickness of $0.57 \mathrm{~mm}$ designed for $17 \times 17$ PWR fuel rod arrays. Pure zirconium tubes with an outside diameter of $12.75 \mathrm{~mm}$ and a $0.95-\mathrm{mm}$ wall thickness were also used as samples in this experiment. The compositions of the two zirconium alloys are provided in Table 1. 
Neutron scattering specimens were cut as 10.0-mm-long rings for scanning vertical to the cladding axis. Hydrogen charging was performed with a new hydriding system recently developed at Oak Ridge National Laboratory (ORNL). The specimens were sealed in a process tube and heated to facilitate hydrogen absorption by the metal. After the hydriding experiment, light microscopy and electron backscatter diffraction (EBSD) were used to examine the hydride morphology, and then direct hydrogen measurements were performed on the hydrided samples using the VHE method (ASTM E 146-83) with a LECO hydrogen analyzer, by which the samples with desired hydrogen concentration were selected for the small angle neutron scattering study. Table 2 lists specific information about each sample examined in this neutron experiment.

The SAINS beam lines have great advantages in performing such hydrogen content measurement. The cold neutron source used for SAINS instruments is very suitable for detecting light elements such as hydrogen, as well as for avoiding the possible diffraction induced from thermal neutron diffraction from the cladding. Also, as the incoherent scattering from hydrogen is a featureless flat scattering for all the directions, the large area detector used by the SAINS instruments will increase the neutron scattering count, improving the statistics of SAINS method. In particular, the SAINS instruments at the High Flux Isotope Reactor (HFIR), with the signature characteristics of high flux and low background combined, are able to detect even very trivial amounts of hydrogen that are present in the cladding material (e.g., 20 ppm, as shown in our experiment).

SAINS experiments were performed on the CG3 and/or CG2 beam line of the HFIR at ORNL [17]. The neutron wavelength was $6 \AA$, with wavelength spread $15 \%$ set using a velocity selector (Mirrotron, Hungary). Scattered neutrons were collected with a $1 \times 1-\mathrm{m}$ two-dimensional position-sensitive detector with $192 \times 256$ pixels. The incoming neutron flux is monitored by a nitrogen-based monitor (Ordela, Inc., Oak Ridge, Tennessee). The SAINS scattered neutron intensity is obtained by summing the intensities measured in all of the area detector pixels and normalizing the results to the monitor detector counts. The size of the collimated incident neutron beam is defined by a neutron-absorbing pinhole aperture plate placed in front and next to the sample. The samples were placed in a holder fixed at the proper height and mounted on a motorized sample stage controlled by an instrument computer, as shown in Fig.1. The distance between the sample and the area detector is about $1.1837 \mathrm{~m}$. The samples were scanned with the incremental sample stage movement of $0.5 \mathrm{~mm}, 1 \mathrm{~mm}$, or $2 \mathrm{~mm}$ (a counting time in tens of seconds per point) so that the distribution of hydrogen could be mapped.

Various Zircaloy-4 cladding samples were scanned along the tube axis for a 63.5-mm-long "tube sample" (see Fig. 2a) or perpendicular to the tube axis for the shorter 10-mm-long "ring samples," as shown in Fig. 2b. Tube sample holders were designed so that the beam line would pass through the center of the horizontally oriented sample and so that the path length would be constant. Sample holders with loaded samples were placed on a motorized stage controlled by an instrument computer. A bird's eye view of the experiment set up is shown in Fig. 2. Samples were scanned with an incremental stage movement of $0.5 \mathrm{~mm}$. After the first neutron scattering run, the same sample was rotated $90^{\circ}$ about the tube axis, and a second scattering run was conducted. After SAINS, ten 1-mm-long short rings were sectioned from the 63.5-mm-long tube sample along the tube axial direction for destructive examination by VHE in order to confirm the hydrogen measurement from SAINS. 


\section{THEORETICAL BACKGROUND FOR SAINS}

Assume that a hydrided sample is placed in a neutron beam with a flux $\mathrm{f}$, and the neutron scattering intensity $\mathrm{I}$ is measured with a neutron detector of area $\mathrm{S}$ at a distance $\mathrm{R}$ from the sample. Considering that both absorption and multiple scattering are low for our application, the intensity I can be given by

$I=f N_{A}\left(M^{H} \sigma^{H}+\sum_{i} \frac{M^{i}}{m^{i}} \sigma^{i}\right) \frac{s}{4 \pi R^{2}}$

where $\mathrm{M}^{\mathrm{H}}$ is the hydrogen mass distributed within sample, $\mathrm{M}^{\mathrm{i}}$ is the mass of other elements with atomic mass $\mathrm{m}^{\mathrm{i}} . \sigma^{\mathrm{H}}$ and $\sigma^{\mathrm{i}}$ are the incoherent scattering cross sections of hydrogen and other elements within the sample, $\mathrm{I}$ is the neutron scattering intensity, $\mathrm{f}$ is the neutron flux beam, and $\mathrm{N}_{\mathrm{A}}$ is Avogadro's constant. For our study on hydrided samples, the only change for various samples is the hydrogen concentration $\left(\mathrm{C}_{\mathrm{h}}=\frac{M^{H}}{\sum_{i} M^{i}}\right)$. Therefore, Eq. (1a) can be simplified as:

$\frac{I}{f}=N_{A} \sum_{i} M^{i}\left(\frac{M^{H}}{\sum_{i} M^{i}} \sigma^{H}+\frac{\sum_{i} \frac{M^{i}}{m^{i}}}{\sum_{i} M^{i}} \sigma^{i}\right) \frac{s}{4 \pi R^{2}}$

and a linear correlation between the normalized scattering neutron intensity $I_{t}=I / f$ and the net hydrogen change in the increments by hydrogen charging $\Delta \mathrm{C}_{\mathrm{h}}$ can be derived,

$\mathrm{I}_{\mathrm{t}}=\mathrm{A} \Delta \mathrm{C}_{\mathrm{h}}+\mathrm{B}$

where $\mathrm{A}$ and $\mathrm{B}$ are fitting constants that can be determined from experiments.

The 10-mm-long ring specimens with various hydrogen concentrations were measured in the radial direction (see Fig 3). A computer controlled motorized specimen stage can be moved perpendicular to the neutron beam. The pass length $\mathrm{L}$ (total wall thickness in beam direction) through the annulus is given by the following equation:

$\mathrm{L}=\left[\mathrm{D}_{\mathrm{o}}{ }^{2} / 4-\left(\mathrm{X}-\mathrm{X}_{\mathrm{o}}\right)^{2}\right]^{1 / 2}-\left[\mathrm{D}_{\mathrm{i}}^{2} / 4-\left(\mathrm{X}-\mathrm{X}_{\mathrm{o}}\right)^{2}\right]^{1 / 2}$, 
where $\mathrm{X}$ is the actual position, $\mathrm{X}_{\mathrm{o}}$ is the middle position of the sample, $\mathrm{D}_{\mathrm{o}}$ is the sample OD, and $D_{i}$ is the sample ID. Figure 3(a) is a schematic top view of neutrons passing through a tube; Fig. 3(b) shows the calculated total wall thickness L across the tube sample in the beam direction.

For samples with much higher hydrogen concentration (>2000 ppm), larger-sized hydride particles (e.g., $\delta$-phase hydrides) might exist that would affect the SAINS data where the scattered intensity would goes beyond the scattering ( $Q$ vector) range of the area detector that may not be collected and thus bias the linear correlation of Neutron Intensity with increasing level of hydrogen concentrations. However, this is beyond the scope of this work. In addition, this SAINS determination of hydrogen concentrations does not attempt to differentiate the scattering from differently shaped hydrides resulting from $\gamma$-phase or $\delta$-phase hydrides.

\section{RESUlts}

\subsection{Microstructural Characterization of Zircaloy-4 Cladding}

\subsubsection{Electron backscatter diffraction}

Electron backscatter diffraction (EBSD) was used to characterize the crystal orientation, grain size, and texture of the as-fabricated Zircaloy-4 cladding. Figures 4(a)-(c) show the inverse pole figure (IPF) coloration mappings and IPFs of the Zircaloy-4 specimens prepared with normal in axial, tangential, and radial directions, respectively. In EBSD, the electron beam is scanned over the sample on a grid of points; at each point, a diffraction pattern is obtained, and the crystal orientation is measured. In the IPF maps, a color is allocated according to the orientation in the inverse pole figure (Fig. 4). The pole figures of the three samples prepared with the plane normal to axial, tangential, and radial directions are shown in Figs. 5(a)-(c). The results of the crystal orientation mapping reveal that our samples are heavily textured. The (0001) pole figure of Fig. 5(c) shows that the cladding has a textured surface, consisting of the preferential orientation of (0001) basal plane with its normal distributed along the radial and transverse direction of the cladding.

For Zircaloy cladding, a thermal treatment (usually $<600^{\circ} \mathrm{C}$ ) is the final processing step; it usually results in full recrystallization (manifested as equiaxed grains) or partial retention of the cold work (manifested as elongated grains along the rolling direction) from the final sizereducing treatment [18-20]. Figures 4(a)-(c) clearly show that our Zircaloy-4 cladding samples consist of elongated grains along the axial direction, indicating that the cladding has partial retention of the cold work during the final stress-relieving treatment for Zircaloy-4 cladding.

\subsubsection{Hydride Morphology}

The hydride morphology of the hydrided Zircaloy-4 cladding was examined with acid etched metallographic samples by light microscopy (Fig. 6). Although the axial gradients in hydrogen 
content were observed in the pre-hydrided samples, our hydriding procedure results in uniform distribution of circumferential hydrides across the cladding wall thickness . Hydride distribution density increases as the hydrogen concentration in the hydrided Zircaloy-4 sample increases. The hydrogen measurements by VHE analysis indicated that the hydrogen contents of the specimens shown in Fig. 6 are from 70 to 320 ppm.

\subsection{SAINS of Ring Samples}

Figure 7 shows the SAINS data of hydrided Zircaloy-4 ring specimens with hydrogen concentration measured by VHE analysis from $15 \mathrm{ppm}$ up to $1100 \mathrm{ppm}$. The neutron scattering intensity is caused by differences in specimen thickness; their hydrogen content increases as the pass length increases, as depicted in Eq. (2). At each specimen position, neutron scattering produced two peaks corresponding to the boundaries of the tube where the neutron beam passes through the largest volume (see Sect. 3 and Fig. 3). The neutron scattering reading from the lowest dip in the curve is the sum of the scattering from the front and back volume of the tube wall thickness. The scaled scattering signal from scanning across the tube provides precise hydrogen concentration. It was observed that the neutron scattering intensity increases proportionally with the hydrogen concentration. For hydrided Zircaloy-4 specimens examined in this work (hydrogen concentration $<1100 \mathrm{ppm}$ ), the intensity increase is linear, in accordance with Eq. (1).

Zircaloy-4 samples comprising Zry4-Ring1 (as-fabricated), Zry4-Ring2, Zry4-Ring3, Zry4-Ring4, Zry4-Ring5, and Zry4-Ring6 were measured by VHE analysis and determined to have approximate hydrogen concentrations of 15 ppm, 80 ppm, 180 ppm, 260 ppm, 580 ppm, 1100 ppm respectively (see Table 2 for details), and the neutron scattering readings from the neutron scattering curve are consistent with these numbers (Fig. 7). The best-fit algorithm for these experimental points gives the linear function:

$\mathrm{I}_{\mathrm{t}}=\mathrm{K} \Delta \mathrm{C}_{\mathrm{h}}+\mathrm{I}_{\mathrm{o}}$

where $\mathrm{K}$ and $\mathrm{I}_{\mathrm{o}}$ are unknown constants, $\mathrm{I}_{t}$ is the normalized sum of all the SAINS counts over the entire area of the 2-D detector, and $\Delta C_{h}$ is the extra hydrogen charged to the specimen. The constant $\mathrm{I}_{\mathrm{o}}$ can be written as

$\mathrm{I}_{\mathrm{o}}=\mathrm{I}_{\mathrm{a}}+\mathrm{I}_{\mathrm{b}}$

where $I_{a}$ is the normalized scattering from the air (neutrons measured without the specimen), and $\mathrm{I}_{\mathrm{b}}$ is the sum over all elements within the as-fabricated specimen, including initial hydrogen (13 ppm for our as-fabricated Zircaloy-4 cladding specimen). We have fitted Eq. (3) with $\mathrm{K}=$ 0.3813 and $\mathrm{I}_{\mathrm{o}}=5$, the result of which is shown in Fig. 8 .

From Eqs. (3) and (4), we obtain

$\Delta \mathrm{C}_{\mathrm{h}}=\mathrm{I}_{\mathrm{t}} / \mathrm{K}-\mathrm{I}_{\mathrm{o}} / \mathrm{K}$ 
Using Eq. (5), we can calculate hydrogen concentration from neutron scattering data.

Fig. 9 shows the SAINS data for hydrided pure $\mathrm{Zr}$ ring specimens. As indicated above, the neutron scattering reading from the lowest dip in the curve is the sum of the scattering from the front and back volume of the tube wall thickness. Similar to Zircaloy-4, we observed that the neutron scattering intensity increase from the hydrided pure zirconium is linear. However, the two neutron scattering peaks corresponding to the boundaries of the tube are not symmetrical about the center, except for the as-fabricated specimen. That indicates that the hydrogen distribution in hydrided pure zirconium rings might not be uniform circumferentially.

The pure zirconium ring samples (PZr-Ring1, PZr-Ring2, PZr-Ring3, and PZr-Ring4) were then sectioned into two half rings, and the hydrogen analysis confirmed a non-uniform hydrogen distribution within the hydrided pure rings (see Table 3). Using the average hydrogen content for each hydrided pure zirconium ring sample, the best-fit algorithm gives the linear function with fitting parameter $\mathrm{K}=0.8552$ and $\mathrm{I}_{0}=15$ for Eq. (3). Fig. 10 shows that the neutron scattering readings from the neutron scattering curve are consistent with directly measured hydrogen content by the VHE method.

\subsection{SAINS of Tube Sample}

Neutron scattering data from two runs (the same sample was rotated $90^{\circ}$ about the tube axis between the two runs) with a hydrided Zircaloy-4 tube sample (Zr4-Tube1) are given in Fig. 11. After the neutron scattering study, ten 1-mm rings were sectioned from the tube sample (space between is roughly $5 \mathrm{~mm}$ ) for direct hydrogen analysis by the VHE method. Fig. 12 is a sectioning diagram showing the 1-mm ring locations, and their hydrogen data are given in Table 3. Using the measured hydrogen content for the ten 1-mm ring samples, the best-fit algorithm gives the linear function with fitting parameter of Eq. (3) $\mathrm{K}=0.3588$ and $\mathrm{I}_{\mathrm{o}}=12$. From Eq. (5), we obtain

$\Delta \mathrm{C}_{\mathrm{h}}=2.7867 \mathrm{I}_{\mathrm{t}}-33$,

where $\mathrm{I}_{\mathrm{t}}$ is neutron scattering intensity from the tube, and $\Delta \mathrm{C}_{\mathrm{h}}$ is the calculated hydrogen content. Using Eq. (6), the distribution of hydrogen along the axis of the hydrided Zircaly-4 tube can be mapped by scaling the number with a factor which is determined by a calibration process with a chemical method. This factor has to be obtained by calibration with a chemical method each time when a different setup is used in the SAINS measurements.

Fig. 13 is a plot of hydrogen content mapped from neutron scattering and the hydrogen content of the ten 1-mm rings measured by the VHE method. The two data sets match very well within errors of the VHE method.

\section{Summary}


The SAINS method has been successfully applied for nondestructive quantitative determination of hydrogen content in hydrided Zircaloy-4 cladding.

Zircaloy-4 cladding was used to produce specimens with controlled hydride content by controlling the initial hydrogen gas pressure in a process tube and the temperature profile of a furnace; targeted hydrogen concentrations ranging from tens of parts per million to a few thousand parts per million were successfully demonstrated.

A very small amount of hydrogen in zirconium-based commercial LWR fuel cladding can be measured very accurately $(\approx 15 \mathrm{ppm})$ in tens of seconds using this method. The hydrogen concentrations determined using the SAINS method on ring-shaped samples have been shown to be consistent with the results of chemical analysis (VHE) within experimental uncertainty.

It was observed that the scattering intensity increases proportionally with the hydrogen charged in the cladding sample examined in this work: at hydrogen concentrations lower than $\approx 1100 \mathrm{ppm}$, the VHE hydrogen content and the SAINS intensity are closely linearly correlated. However, at higher hydrogen concentrations (>1100 ppm) multiple scattering effects begin to influence the measured SAINS intensity profile. To evaluate this effect, a SAINS study of samples prepared with very high hydrogen concentrations $(>4000 \mathrm{ppm})$ is in progress; these results will be reported in the near future.

\section{Acknowledgments}

This research was sponsored by the Laboratory Directed Research and Development (LOIS-6502) Program of Oak Ridge National Laboratory, managed by UT-Battelle, LLC, for the U.S. Department of Energy under Contract No. DE-AC05-00OR22725), and EBSD through a user project supported by ORNL's Center for Nanophase Materials Sciences (CNMS), which is sponsored by the Scientific User Facilities Division, Office of Basic Energy Sciences, U.S. Department of Energy. Beamline CG3 is supported by the Office of Biological and Environmental Research of the U.S. Department of Energy Research through ORNL Center for Structural Molecular Biology. The High Flux Isotope Reactor and beamline CG2 of ORNL was sponsored by the Scientific User Facilities Division, Office of Basic Energy Sciences, U.S. Department of Energy. We are grateful to Dr. Jeremy Busby for his technical inputs and stimulating discussions. 


\section{REFERENCES}

1. V. A. Somenkov and S. S. Shil'stein, "Phase Transitions of Hydrogen in Metals," Progress in Materials Science 24, 267-335 (1979).

2. J. Chevallier and M. Aucoturier, Annual Review of Materials Science 18, 219-56 (1988).

3. A. M. Garde et al., "Advanced Zirconium Alloy for PWR Application," Journal of ASTM International 7, No. 9, doi:10.1520/JAI103030.

4. V. Chabretou et al., "Ultra Low Tin Quaternary Alloys PWR Performance-Impact of Tin Content on Corrosion Resistance, Irradiation Growth, and Mechanical Properties," Journal of ASTM International 8, No. 5, doi:10.1520/JAI103013.

5. J. V. Cathcart et al., Zirconium Metal-Water Oxidation Kinetics IV. Reaction Rate Studies, ORNL/NUREG-17, Oak Ridge National Laboratory, Oak Ridge, Tenn., August 1977.

6. Y. Yan et al., High Temperature Oxidation of Irradiated Limerick BWR Cladding, NUREG/CP-0176, 353-72, May 2002.

7. F. Nagase, T. Otomo, and H. Uetsuka, "Oxidation Kinetics of Low-Sn Zircaloy at the Temperature Range from 773 to 1571K," Journal of Nuclear Science and Technology 40, 213-19 (2003).

8. M. Billone et al., Cladding Embrittlement during Postulated Loss-of-Coolant Accidents, NUREG/CR-6967, ANL-07/04, Argonne National Laboratory, Argonne, Ill., July 2008.

9. J. C. Brachet et al., "Influence of Hydrogen Content on the $\alpha / \beta$ Phase Transformation Temperature and on the Thermal-Mechanical Behavior of $\mathrm{Zy}-4, \mathrm{M} 4(\mathrm{ZrSnFeV})$, and M5 ${ }^{\mathrm{TM}}(\mathrm{ZrNbO})$ Alloys During the First Phase of LOCA Transient," pp. 673-701 in Zirconium in the Nuclear Industry, ASTM STP 1423, G. D. Moan and P. Rudling, eds., American Society for Testing and Materials, 2002.

10. Y. Yan, T. Burtseva, M. Billone, "High-temperature Steam-oxidation Behavior of Zr-1Nb Cladding Alloy E110," Journal of Nuclear Materials 393, No. 3, 433-48 (2009).

11. T. Cheng et al., "Oxidation of Fuel Cladding Candidate Materials in Steam Environments at High Temperature and Pressure," Journal of Nuclear Materials 427, Nos. 1-3, 396-400 (2012).

12. Y. Yan et al., "Post Quench Ductility Evaluation of Zircaloy-4 and Select Iron Alloys under Design Basis and Extended LOCA Conditions," Journal of Nuclear Materials, to be published.

13. R. Yasuda et al., "Application of Hydrogen Analysis by Neutron Imaging Plate Method to Zircaloy Cladding Tubes," Journal of Nuclear Materials 320, 223-30 (2003).

14. M. Grosse et al., "Quantitative Determination of Absorbed Hydrogen in Oxidised Zircaloy by Means of Neutron Radiography," Nuclear Instruments and Methods in Physics Research Section A: Accelerators, Spectrometers, Detectors and Associated Equipment 566, No. 2, 739-45 (2006).

15. P. Von Der Hardt and H. Rotter, eds., Neutron Radiography Handbook, D. Reidel Publishing Co., Dordrecht, Holland, 1981 p. 58.

16. E. H. Lehmann, P. Vontobel, and N. Kardjilov, "Hydrogen Distribution Measurements by Neutrons," Applied Radiation and Isotopes 61, No. 4, 503-09 (2004).

17. W. T. Heller, V. S. Urban, G. W. Lynn, K. L. Weiss, H. M. O'Neill, S. V. Pingali, S. Qian, K. C. Littrell, Y. B. Melnichenko, M. V. Buchanan, D. L. Selby, G. D. Wignall, P. D. Butler and D. A. Myles, "The Bio-SANS instrument at the High Flux Isotope Reactor of Oak Ridge National Laboratory “ J. Appl. Cryst. (2014). 47, 1238-1246

18. C. Lemaignan, A. T. Motta, and B. R. T Frost, "Zirconium Alloys in Nuclear Applications," Nuclear Materials, R.W. Cahn, P. Haasen, and E. J. Kramer, eds., Materials Science and Technology series, 1994, John Wiley and Sons, New York, 1994, pp. 1-52.

19. L. Moulin, S. Reschke, and E. Tenckhoff, "Correlation Between Fabrication Parameters, Microstructure, and Texture in Zircaloy Tubing," Zirconium in the Nuclear Industry: Sixth International Symposium, ASTM STP 824, 1984, pp. 225-43. 
20. D. L. Baty, "Deformation Characteristics of Cold-worked and Recrystallized Zircaloy-4 Cladding," Zirconium in the Nuclear Industry: Sixth International Symposium, ASTM STP 824, 1984, pp. 306 39. 
Table 1. Alloy compositions in percent mass fraction

\begin{tabular}{|l|c|c|c|c|c|c|c|c|c|}
\hline \multicolumn{1}{|c|}{ Alloy } & Fe & Hf & Sn & $\mathbf{C r}$ & $\mathbf{A l}$ & $\mathbf{T i}$ & $\mathbf{S i}$ & $\mathbf{O}$ & $\mathbf{Z r}$ \\
\hline Zircaloy-4 Cladding & 0.22 & - & 1.26 & 0.10 & - & - & 0.01 & 0.130 & balance \\
\hline Zr tube & 0.09 & 0.35 & - & 0.01 & 0.01 & 0.02 & - & 0.114 & balance \\
\hline
\end{tabular}

Note: These data were analyzed compositions by combustion methods.

Table 2. Samples used in neutron experiment

\begin{tabular}{|l|l|c|c|}
\hline Sample ID & Materials & $\begin{array}{c}\text { Sample } \\
\text { Length } \\
\text { (mm) }\end{array}$ & $\begin{array}{c}\text { Hydrogen content measured by VHE from two } \\
\text { 1-mm-long rings at two ends of the 10-mm specimen } \\
\text { (ppm) }\end{array}$ \\
\hline Zry4-Ring1 & Zircaloy-4 & 10 & $\approx 15$ \\
\hline Zry4-Ring2 & Zircaloy-4 & 10 & $\approx 80$ \\
\hline Zry4-Ring3 & Zircaloy-4 & 10 & $\approx 180$ \\
\hline Zry4-Ring4 & Zircaloy-4 & 10 & $\approx 260$ \\
\hline Zry4-Ring5 & Zircaloy-4 & 10 & $\approx 580$ \\
\hline Zry4-Ring6 & Zircaloy-4 & 10 & $\approx 1100$ \\
\hline PZr-Ring1 & Pure Zr & 10 & $\approx 20$ \\
\hline PZr-Ring2 & Pure Zr & 10 & $\approx 110$ \\
\hline PZr-Ring3 & Pure Zr & 10 & $\approx 320$ \\
\hline PZr-Ring4 & Pure Zr & 10 & $\approx 440$ \\
\hline
\end{tabular}


Table 3. Hydrogen content of a hydrided Zircaloy-4 tube sample Zr4-Tube1 measured by VHE. The ten samples were sectioned from a 3-in.-long tube at different axial locations.

\begin{tabular}{ccc}
\hline Sample ID & $\begin{array}{c}\text { Hydrogen content } \\
\text { (ppm) }\end{array}$ & $\begin{array}{c}\text { LECO Instrument Error } \\
\text { (ppm) }\end{array}$ \\
\hline Zr4-Tube1-A & 193 & $\leq 15$ \\
Zr4-Tube1-B & 218 & $\leq 15$ \\
Zr4-Tube1-C & 241 & $\leq 15$ \\
Zr4-Tube1-D & 259 & $\leq 15$ \\
Zr4-Tube1-E & 301 & $\leq 15$ \\
Zr4-Tube1-F & 361 & $\leq 15$ \\
Zr4-Tube1-G & 397 & $\leq 15$ \\
Zr4-Tube1-H & 433 & $\leq 15$ \\
Zr4-Tube1-I & 442 & $\leq 15$ \\
Zr4-Tube1-J & 447 & $\leq 15$ \\
\hline
\end{tabular}




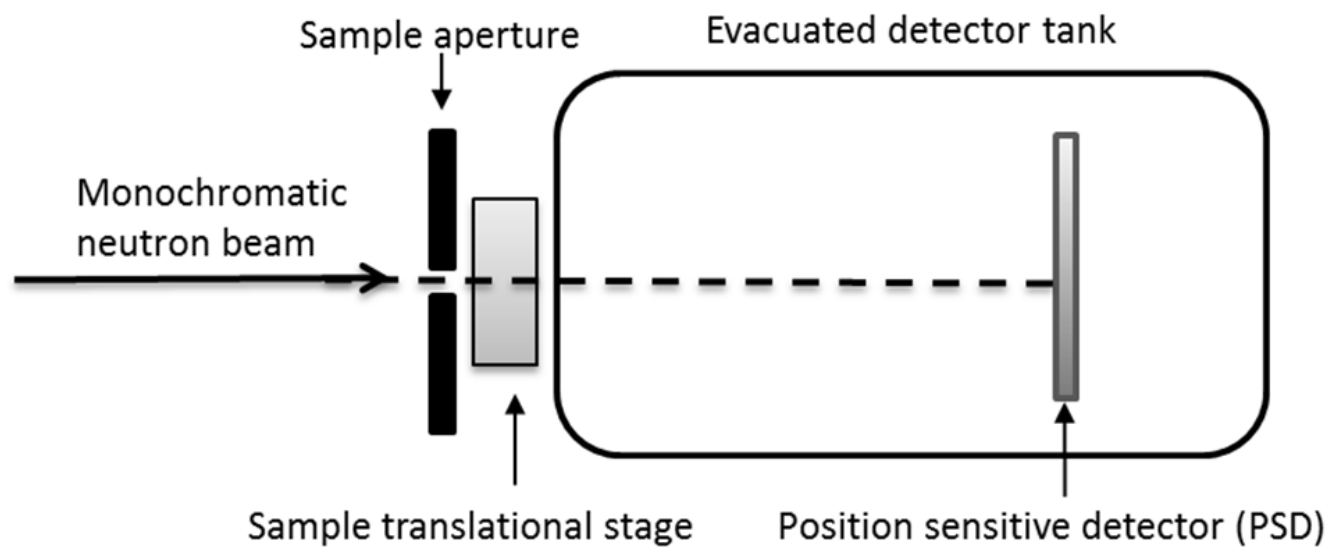

Fig. 21. Neutron scattering instrument configuration.

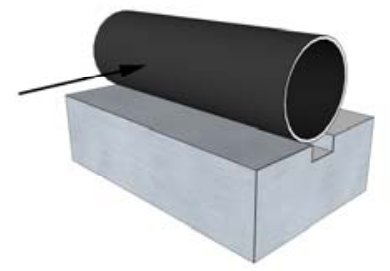

(a)

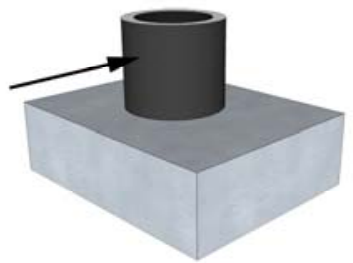

(b)

Fig. 2. Schematic of experiment set up with (a) tube sample and (b) ring sample. 


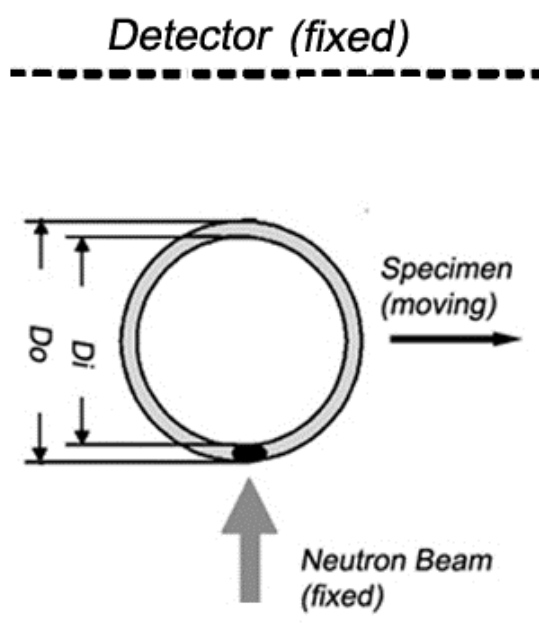

(a)

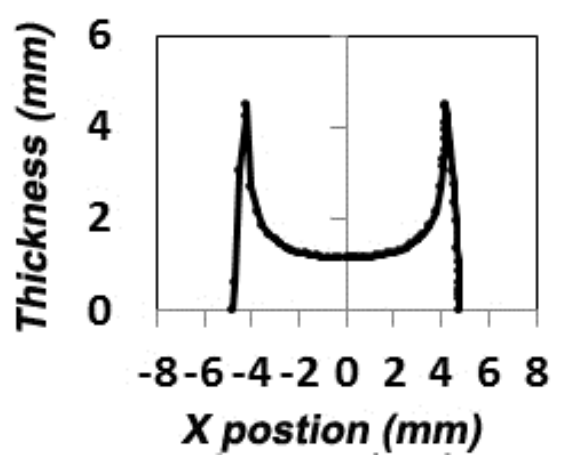

(b)

Fig. 3. (a) Schematic top view of neutrons passing through a tube; (b) calculated total wall thickness $L$ across the tube sample in beam direction.

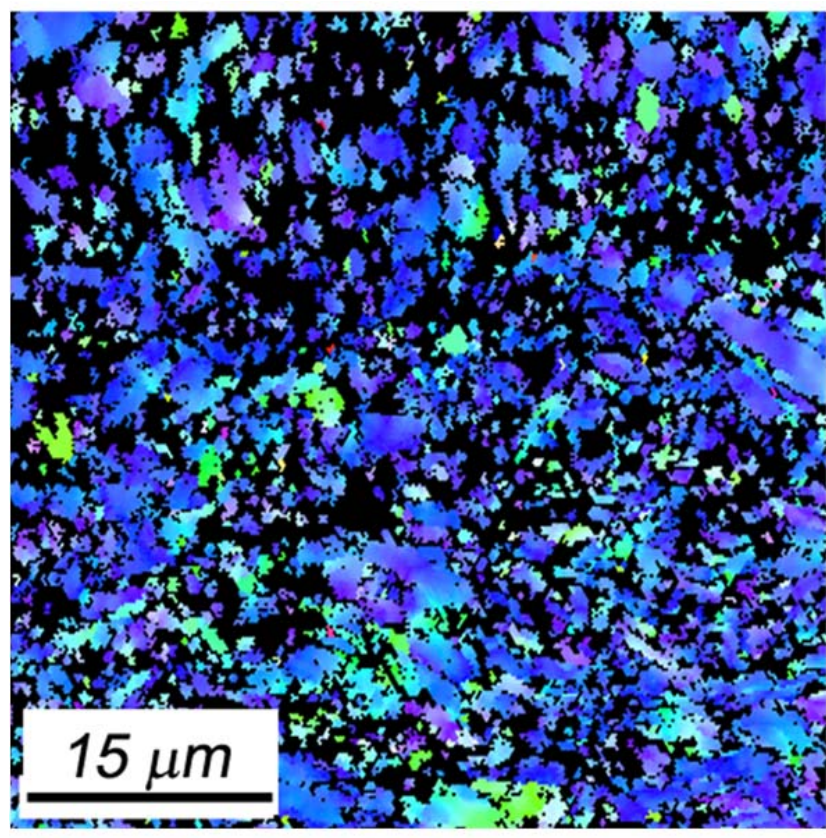

IPF in axial direction (normal)
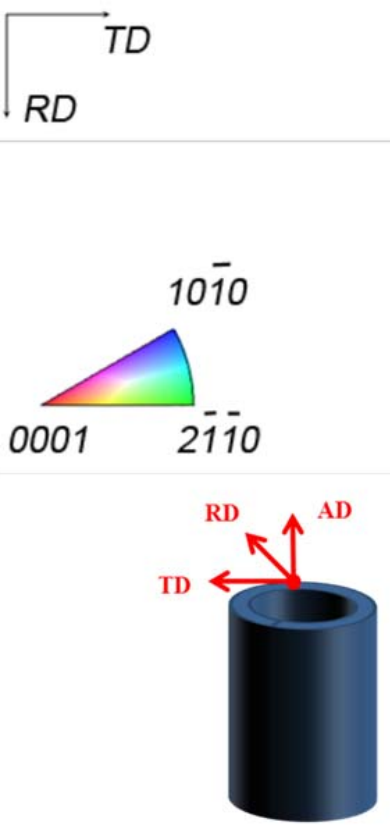

(4a) 

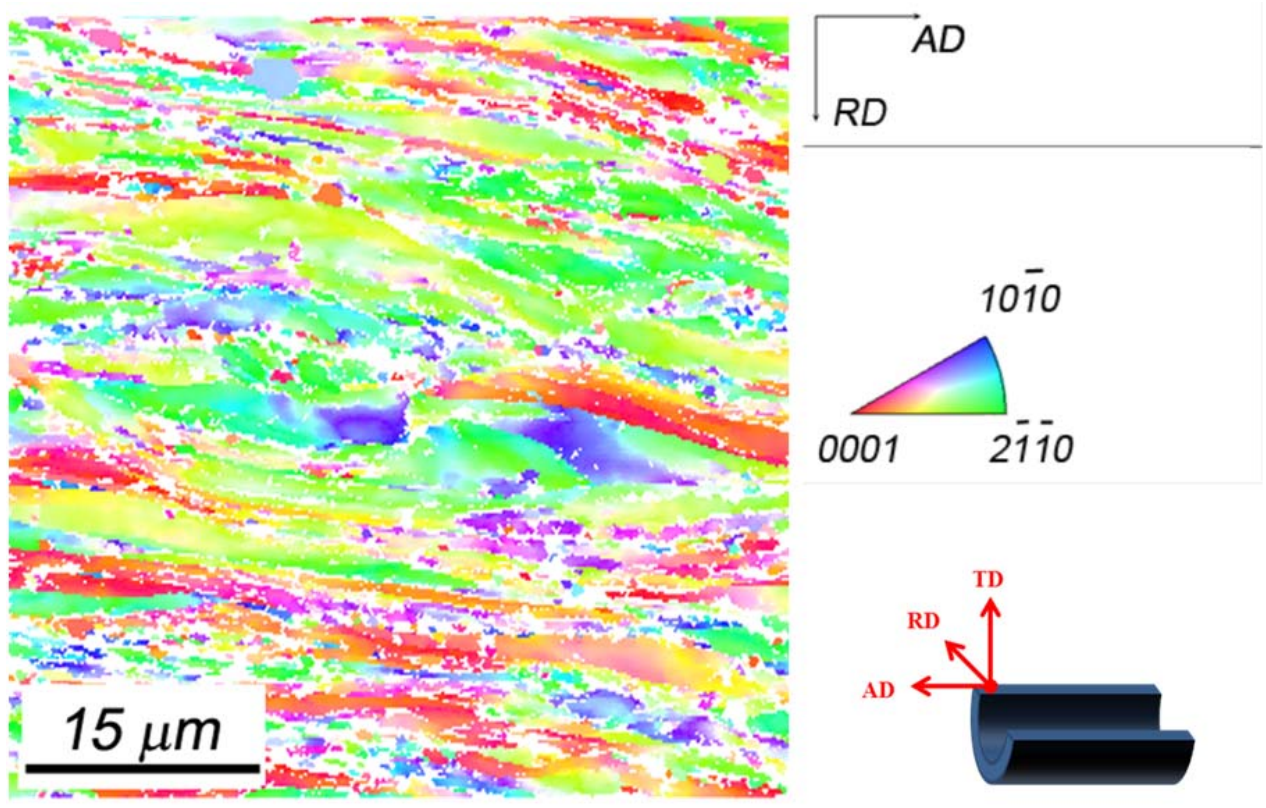

IPF in tangential (normal)

(4b)

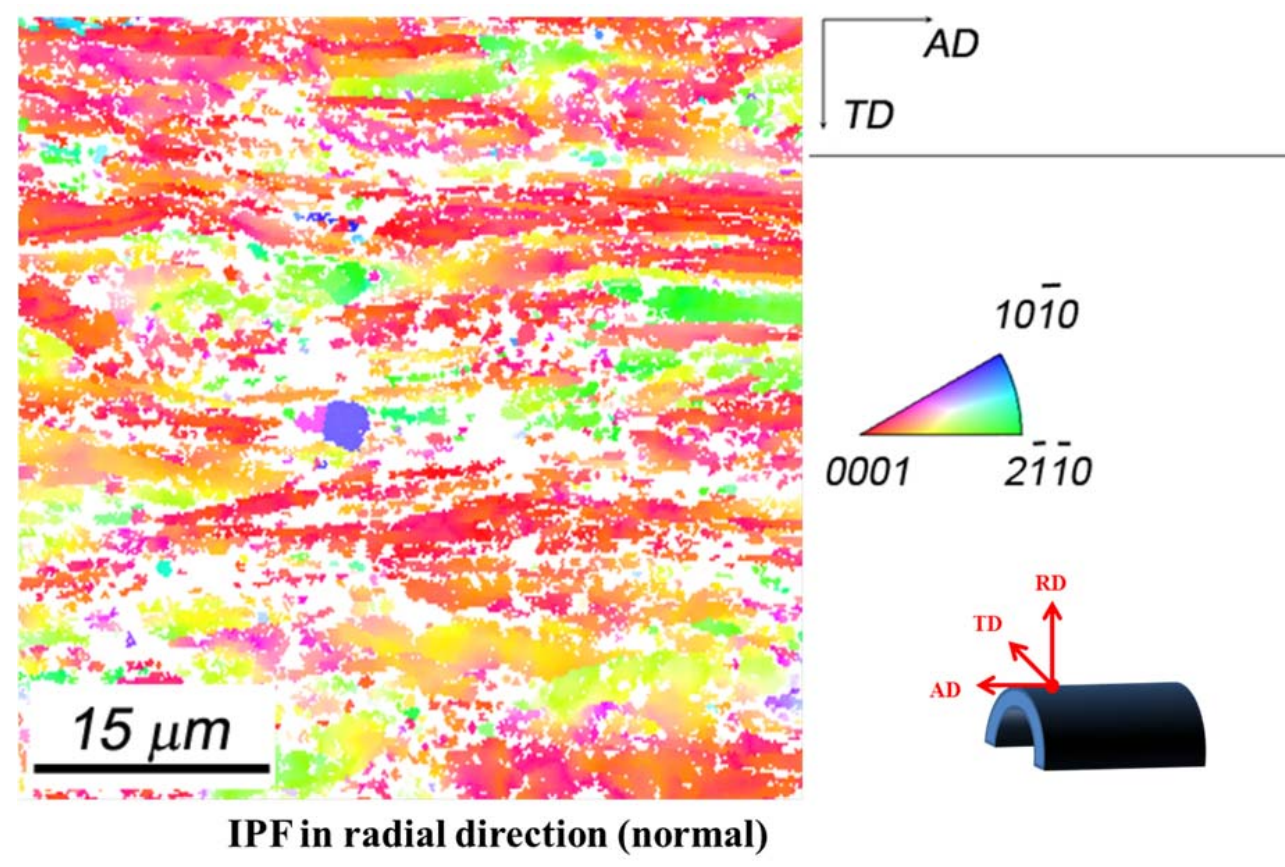

(4c)

Fig. 4. Inverse pole figure mappings of the Zircaloy-4 specimens prepared in (a) axial, (b) tangential, and (c) radial directions, respectively. TD, AD and RD mean axial direction, tangential direction, and radial direction, respectively. 

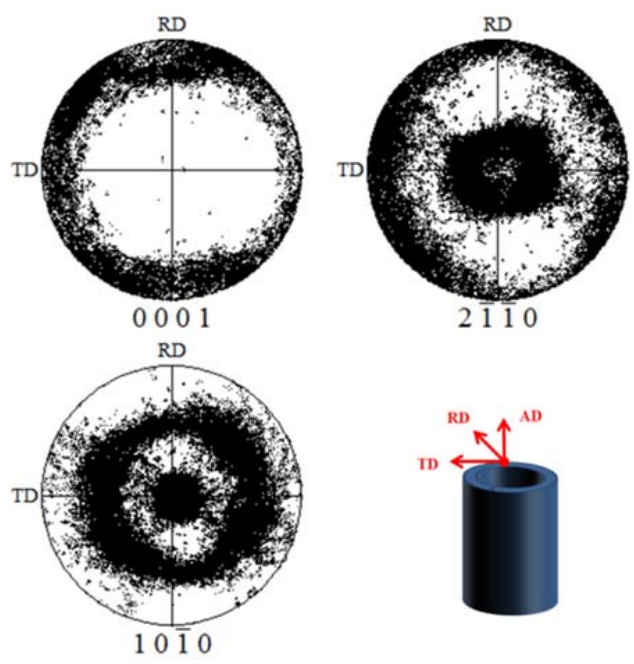

(a)
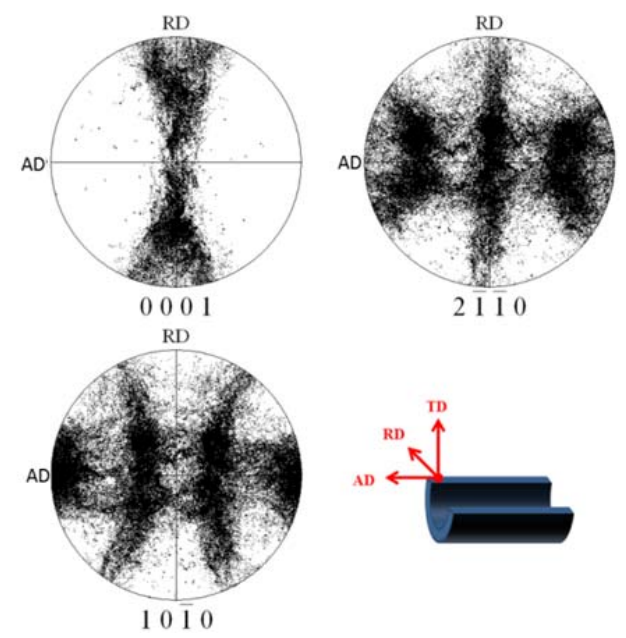

(b)
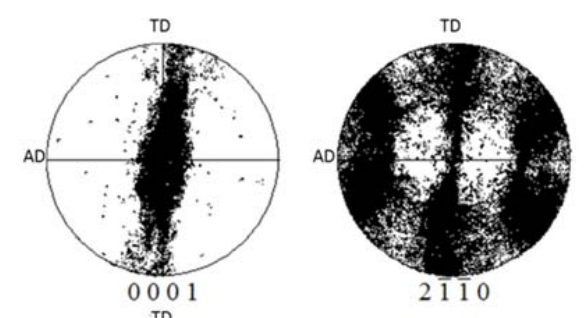

(c)
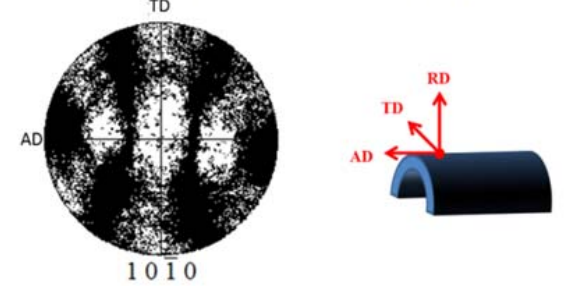

Fig. 5. Pole figures of he three samples prepared with normal in (a) axial, (b) tangential, and (c) radial directions are shown in Figs. 5(a)-(c). 


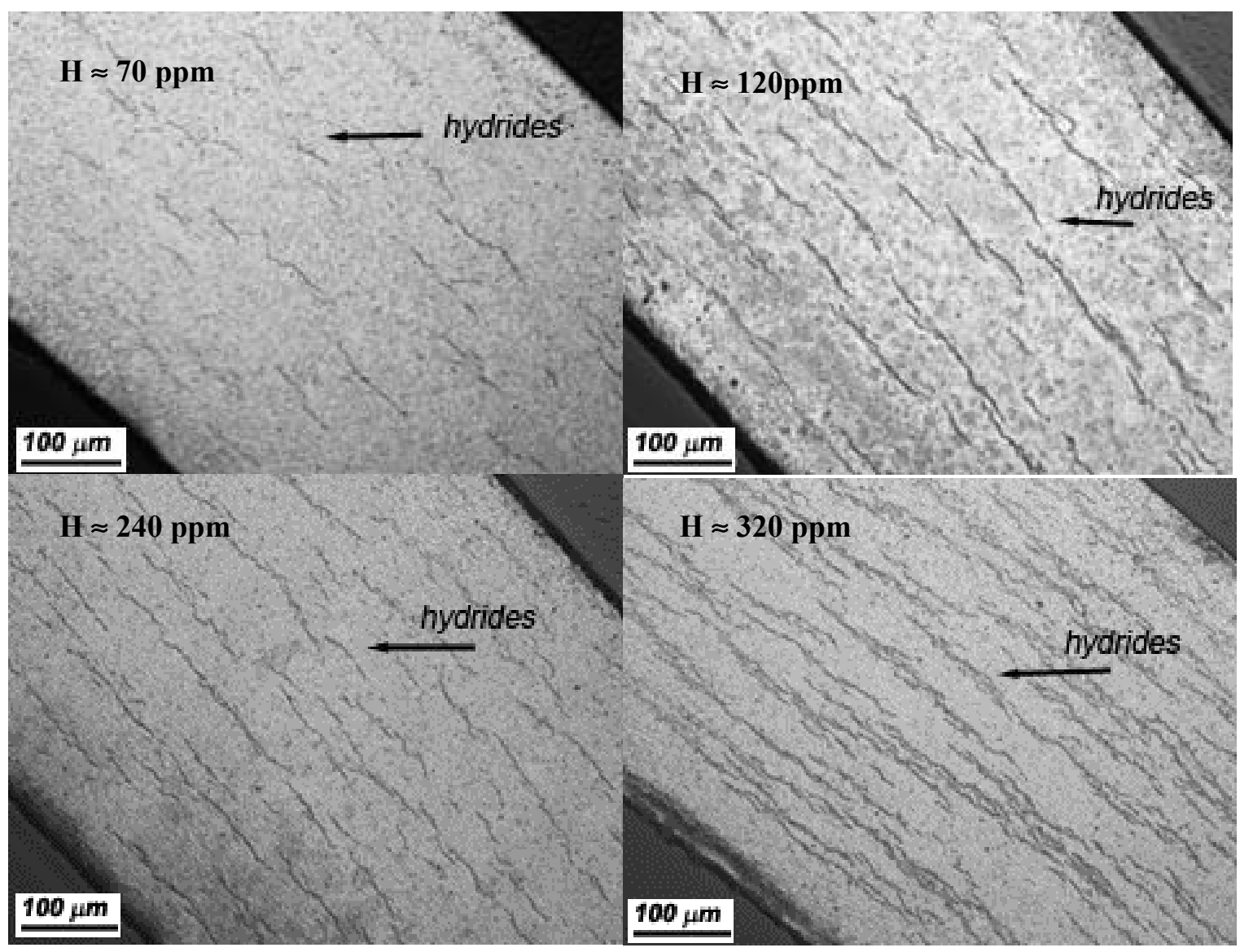

Fig. 6. Optical micrographs showing hydride distributions in hydrogen-charged Zircaloy-4 samples in this study. The given hydrogen concentrations are an average for the cladding at the location examined. 


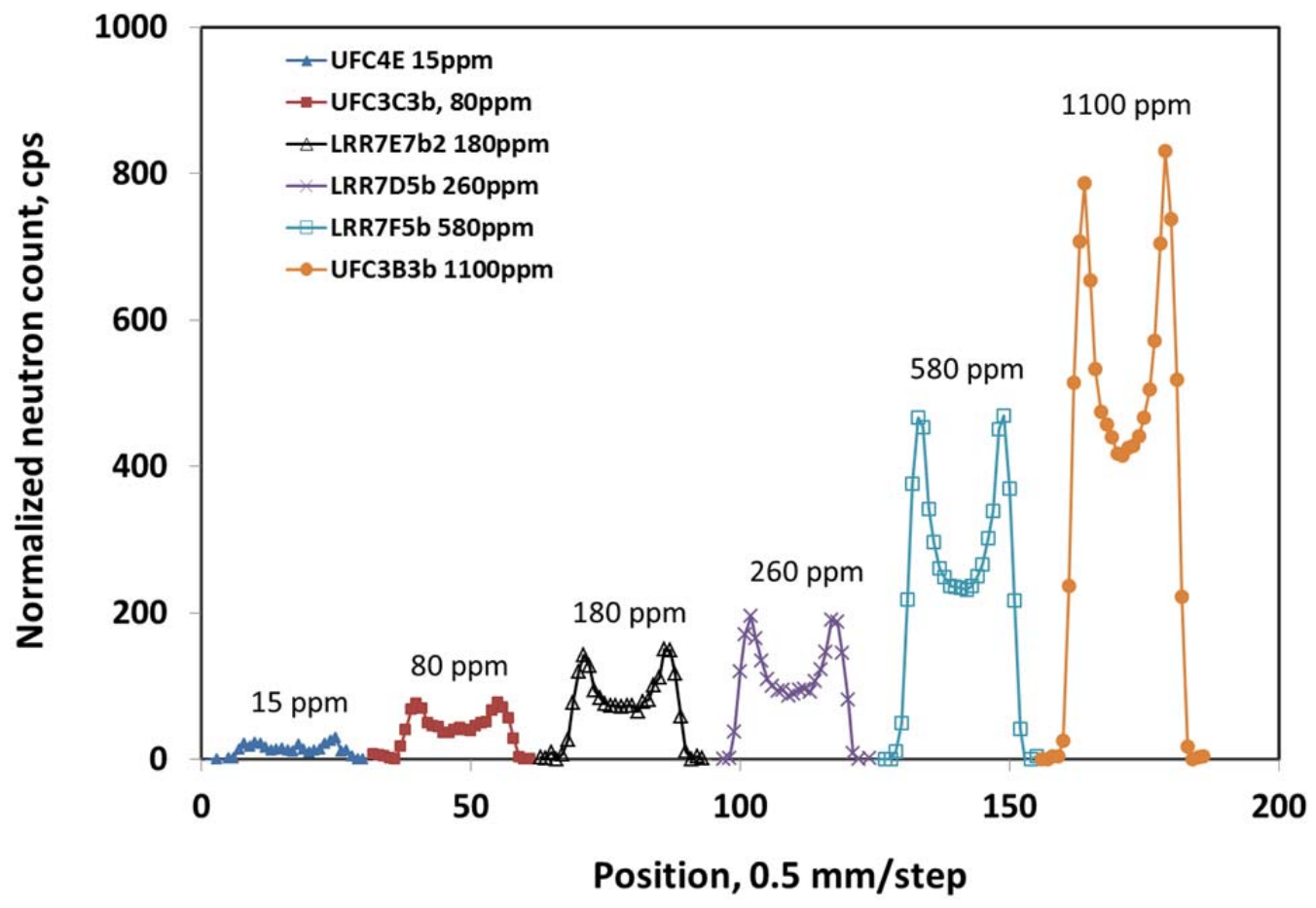

Fig. 7. Net neutron counts of Zircaloy-4 ring samples. The samples are arranged by ascending hydrogen concentration.

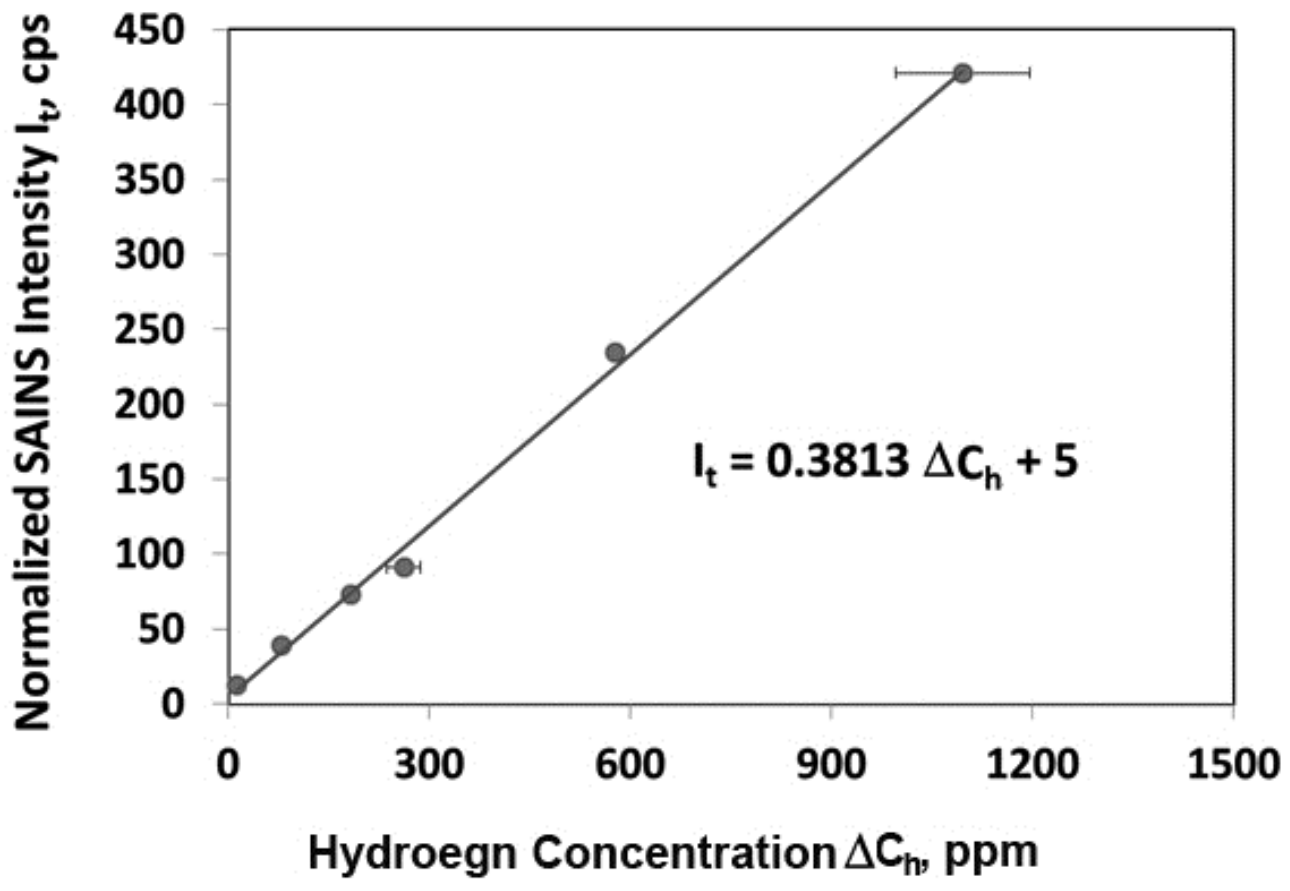

Fig. 8. Neutron scattering intensity as a function of hydrogen concentration for hydrided Zircaloy-4 cladding. 


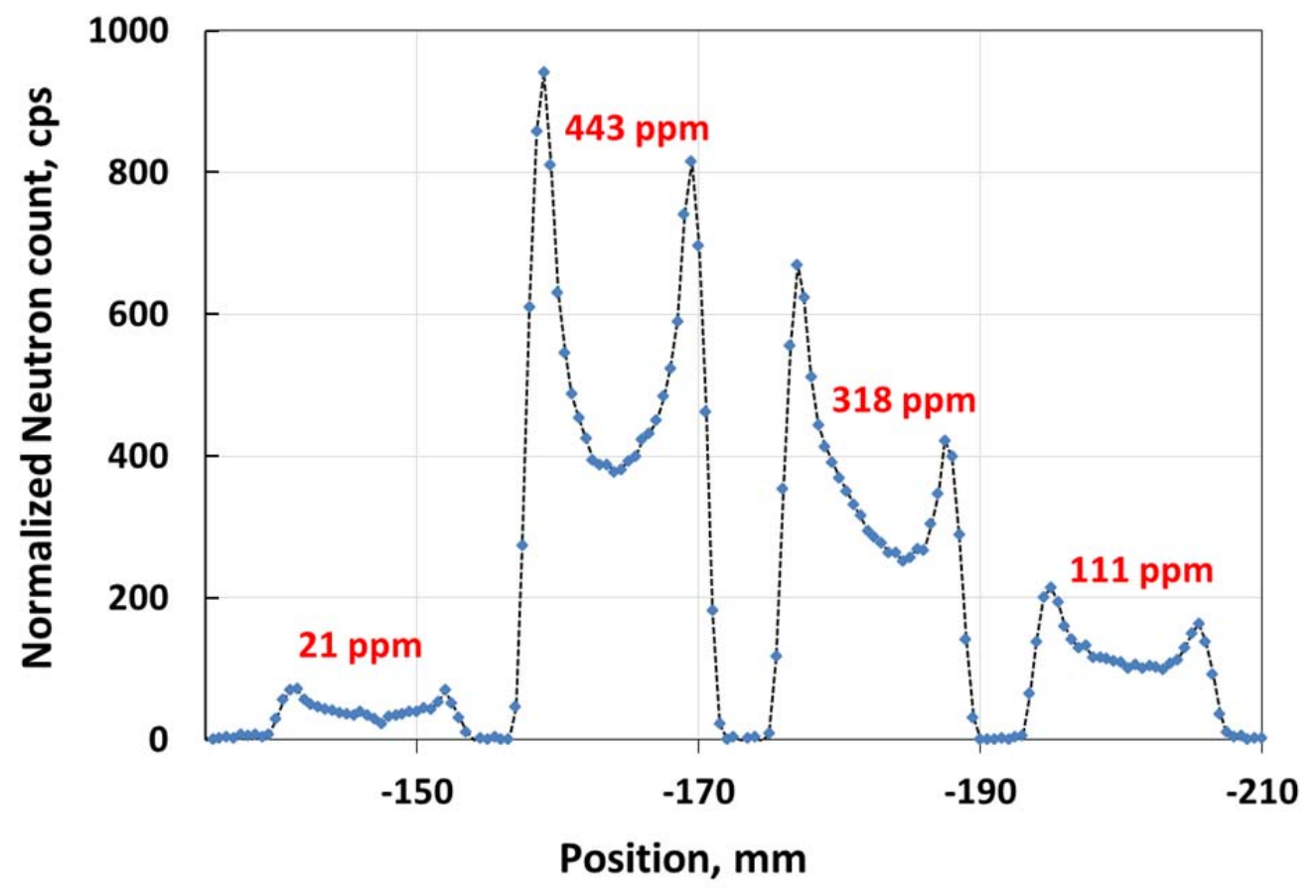

Fig. 9. Net neutron count with added average background of pure zirconium ring samples. 


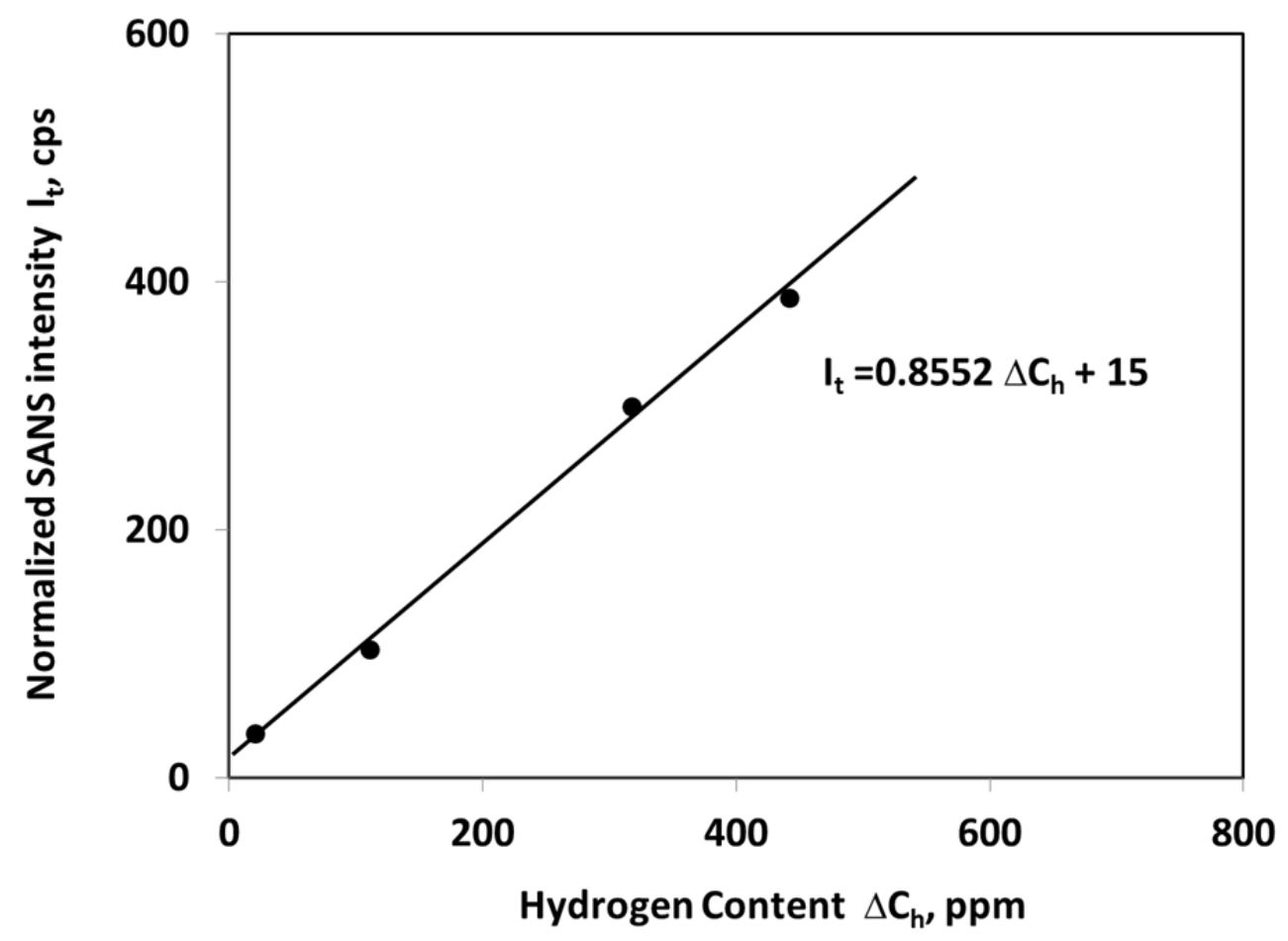

Fig. 10. Neutron scattering intensity as a function of hydrogen concentration for hydrided pure zirconium cladding. 


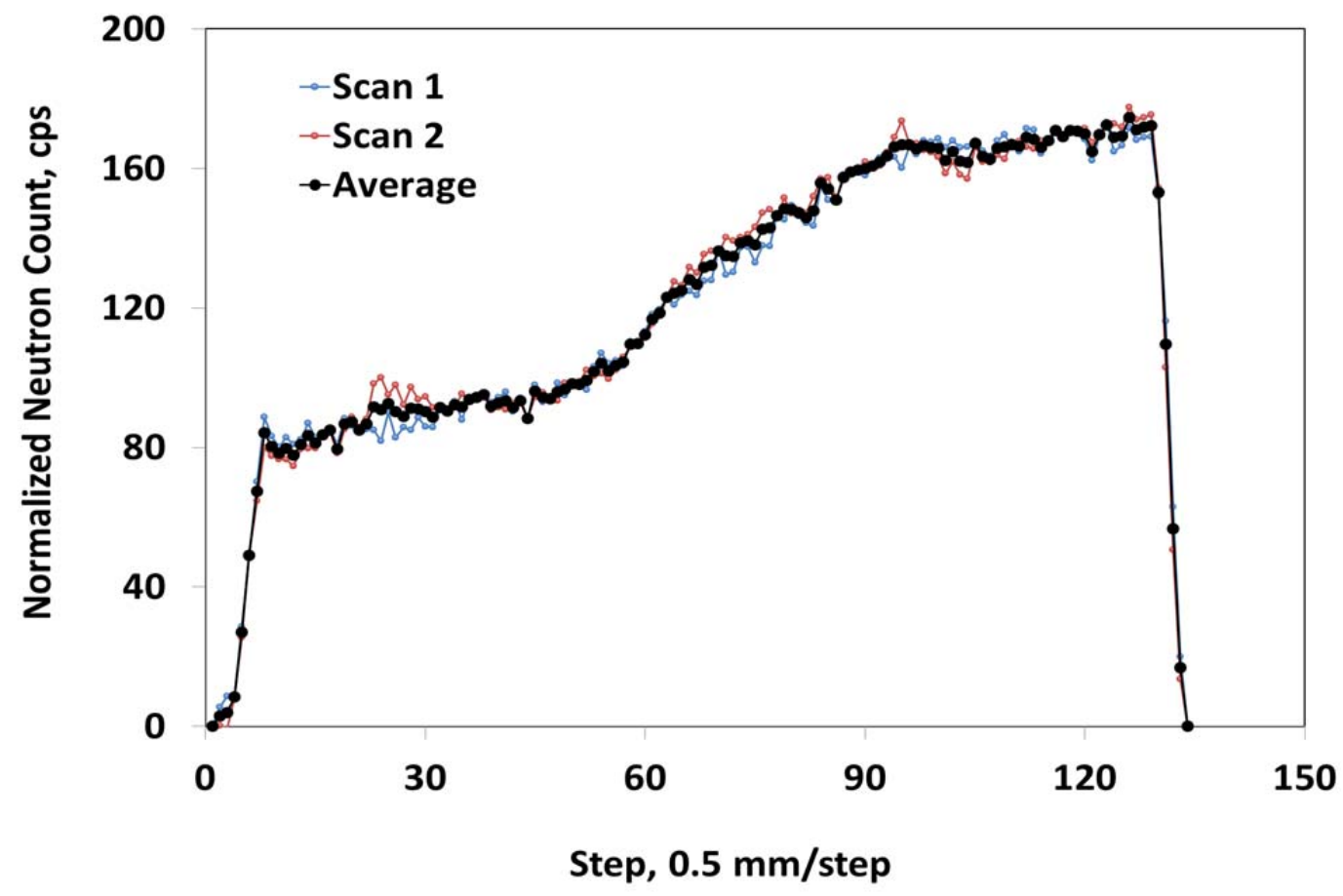

Fig. 11. Plot of a 63.5-mm-long Zircaloy-4 sample. Scan 2 data from the same sample rotated $90^{\circ}$ about the tube axis.

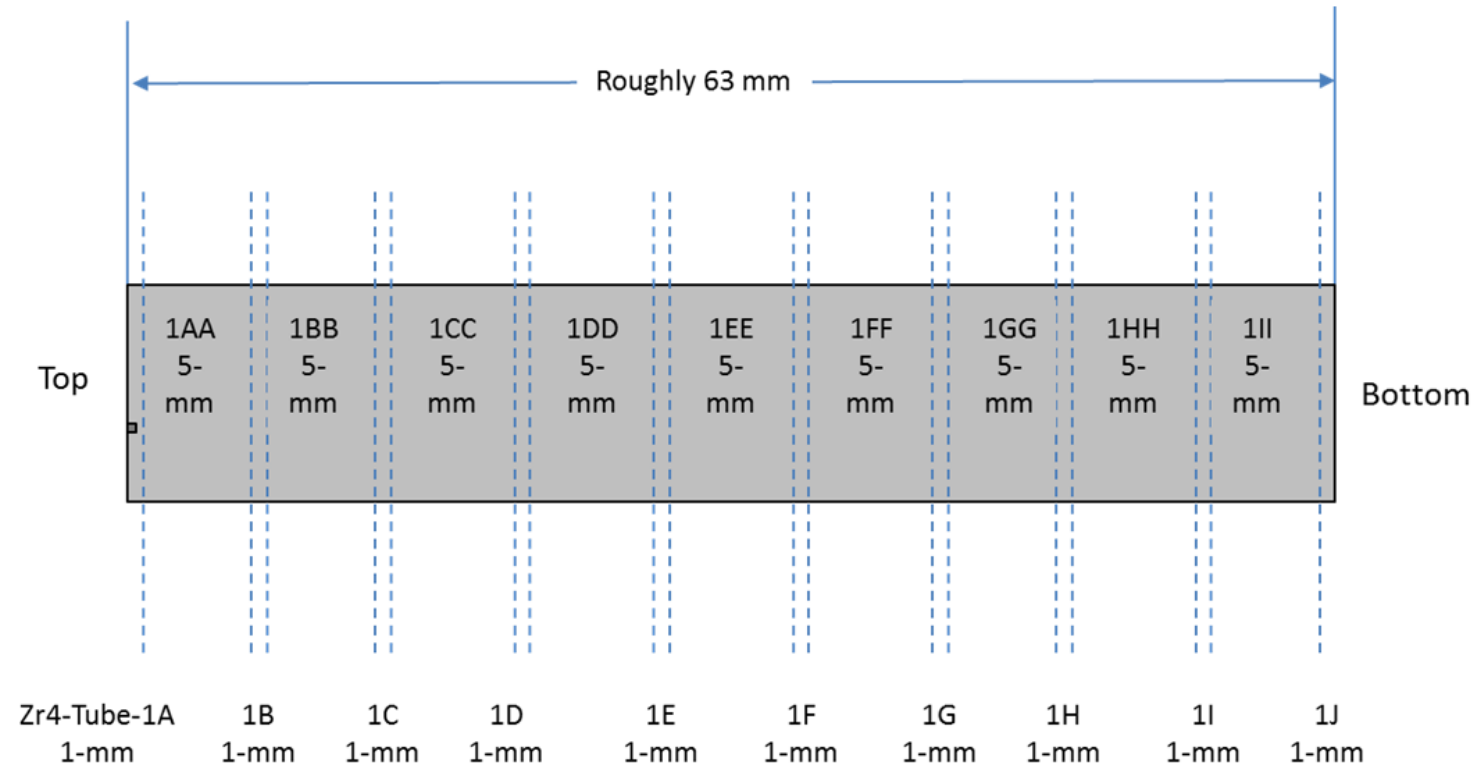

Fig. 12. Sectioning diagram for neutron scattering sample Zr4-Tube1. The ten 1-mm rings (Zr4-Tube-1A to Zr4-Tube-1J) are sectioned for hydrogen analysis. 


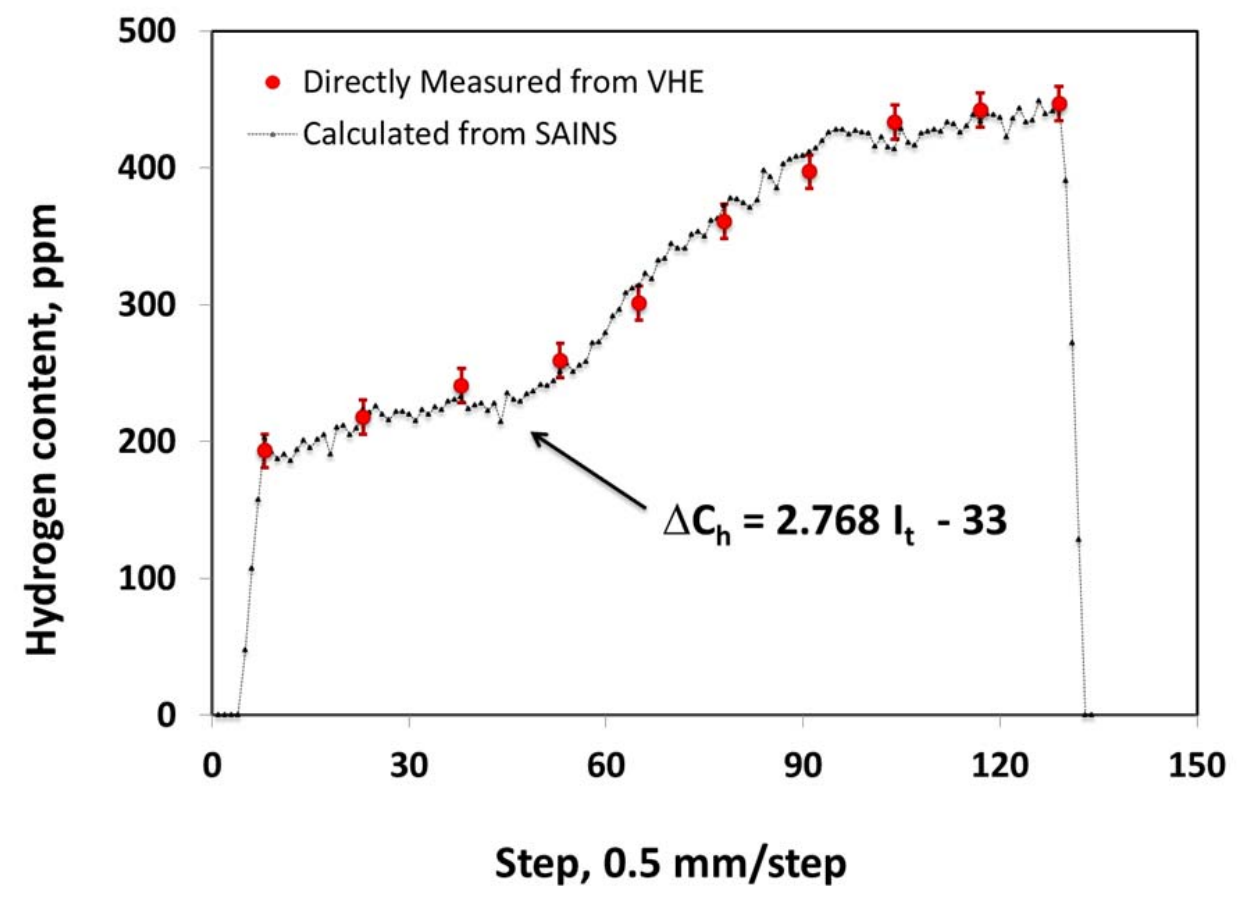

Fig. 13. Comparison between the VHE-measured and the SAINS-calculated hydrogen concentration. 